\title{
Calibration of an Accelerometer
}

\section{Kimura Hitoshi*}

Department of Mechanical and Control Engineering, Tokyo Institute of Technology, Japan

\begin{abstract}
Conventional calibration method of an accelerometer does not take account of the direction gap between the package and the internal sensors. The gap causes sensitivity error and crosstalk of other axis acceleration. These errors prevent to calculate accurate input acceleration. This study proposes a new calibration method which provides actual sensor directions and individual sensitivities.
\end{abstract}

\section{Introduction}

Accelerometers have been widely used in the last 10 years. Now they are embedded in smartphones, controllers of game machines and so on. This article regards an accelerometer as a package which is embedded several internal acceleration sensors. Recently, an MEMS 3-axis accelerometer is popular for industrial use.

The calibration methods of the accelerometers are defined in industrial standards, represented by ISO $[1,2]$. These methods are based on an assumption that the actual sensor directions are aligned with the package, for example, $\mathrm{x}$-direction sensor is parallel to the $\mathrm{x}$-direction of the package. In actual fact, this assumption is not guaranteed because there is a direction gap between any sensors and the package caused by manufacturing error. This causes calculation error of the input acceleration.

Following discussion is a simple example of calculation error of
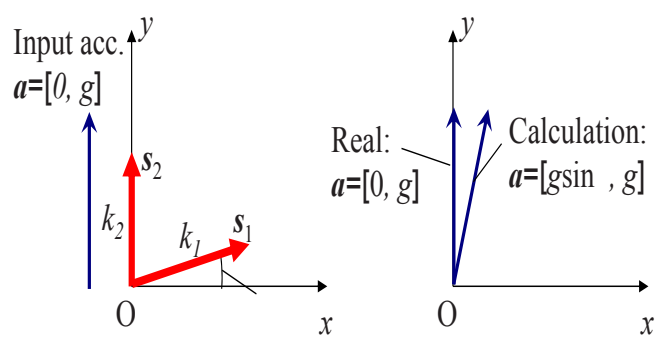

Figure 1: Calculation error of the input acceleration caused by inclination of the sensor direction.

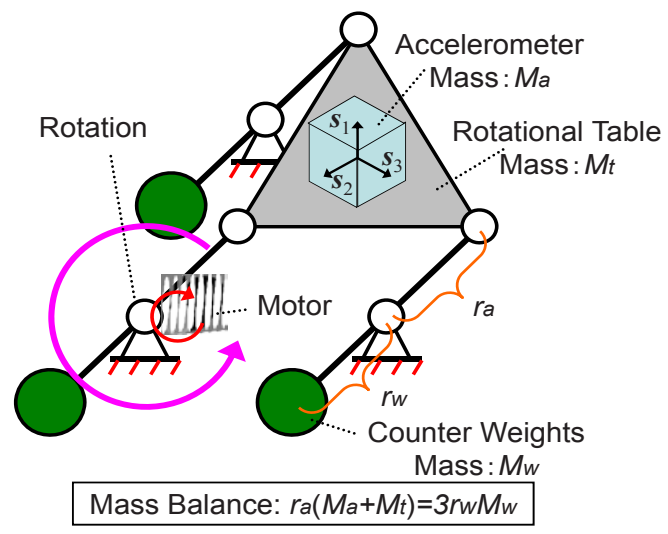

Figure 2: Exciter devices with parallel linkage. the input acceleration. For simplicity, this discussion deals with two dimensional spaces. There are two sensors in $x y$-plane. The sensors for $x$-direction and for $y$-direction are defined as sensor 1 and sensor 2 respectively. Hypothesizing that sensor 1 is inclined at the angle $\theta$ as drawn in figure 1. The sensitivities of the two sensors are $k_{1}$ and $k_{2}$ $\left[\mathrm{V} /\left(\mathrm{ms}^{-2}\right)\right]$ respectively. Output voltage vector (for simple expression) $v_{\text {out }}=\left[k_{1} \sin \theta, k_{2}\right][\mathrm{V}]$ of the sensor when the input acceleration vector $\mathrm{a}=[0,1]\left[\mathrm{ms}^{-2}\right]$. If the calculation of the input acceleration is based on the hypothesis that the sensor directions are aligned with the package direction, the result becomes $a=[\sin \theta, 1]\left[\mathrm{ms}^{-2}\right]$. This might be an intuitive example for calculation error of the input acceleration caused by the sensor direction gap. In addition, it is obvious that exact $k_{1}$ and $k_{2}$ are necessary to calculate the input acceleration.

The above mentioned discussion is quite simple and obvious; nevertheless, the measurement of the internal sensor inclination was not easy. Moreover, offset voltage change is one of sticky problems. The accelerometer outputs offset voltages as standard voltages in general and the voltages always slightly change. For the purpose of removing the offset voltages, constant input acceleration is not effective. That is
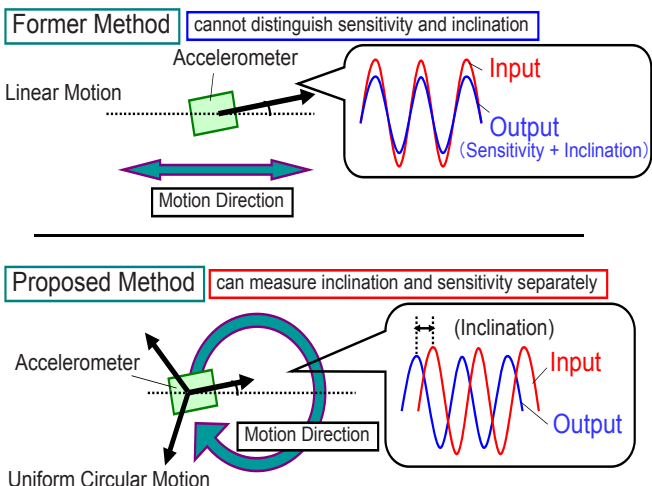

Sensor Posture: Constant

Figure 3: Characteristics of calibration methods.

*Corresponding author: Kimura Hitoshi, Department of Mechanical and Control Engineering, Tokyo Institute of Technology, Japan, E-mail: kimura@mech.titech.ac.jp

Received August 03, 2012; Accepted August 06, 2012; Published August 08, 2012

Citation: Hitoshi K (2012) Calibration of an Accelerometer. J Appl Mech Eng 1:e105. doi:10.4172/2168-9873.1000e105

Copyright: (c) 2012 Hitoshi K. This is an open-access article distributed under the terms of the Creative Commons Attribution License, which permits unrestricted use, distribution, and reproduction in any medium, provided the original author and source are credited. 

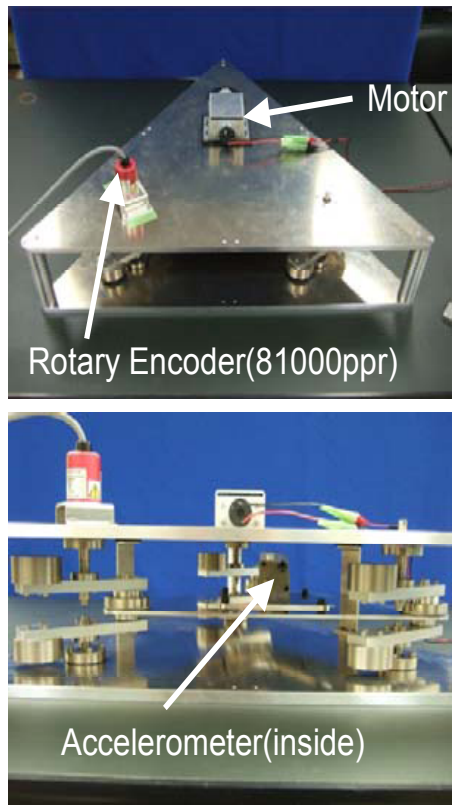

Figure 4: Exciter mechanisms with parallel linkage.

\section{$\bigotimes_{i}$ : The plane including actual sensor of $i$ axis}

Measured Planes for Peak Angle $\nabla_{i} n_{i m}:$ Normal vector of $\nabla_{i}$ plane in $m^{\text {th }}$ measurement

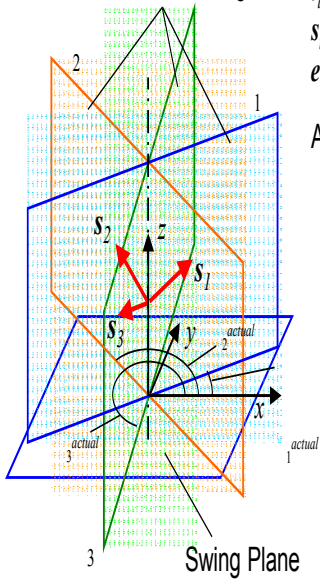

$s_{i}:$ Actual sensor vector of $i$ axis

$e_{i}:$ Base vector of I axis (ideal sensor direction)

Accelerometer Package

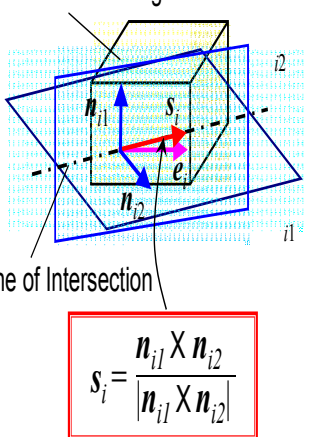

Figure 5: Sensor including planes and actual sensor direction.

why ISO does not recommend using gravity but artificial sine waves or chopping waves as the input acceleration for the calibration.

To find actual sensor direction, following conditions are required: 1) Reliable input acceleration with high accuracy, 2) Aligning the acceleration direction so as to maximize the sensor output voltage. It is not easy to realize these conditions in general. Almost all manufacturers allow a certain inclination of the sensor direction and call the error as crosstalk at the present stage. However, if we have the data of actual sensor directions and individual sensitivities, we can calculate accurate input acceleration vector using a coordinate transformation. In addition, it is possible to calculate the accurate acceleration from an accelerometer which has neither orthogonal sensor directions nor equal sensitivities. Such accurate acceleration data obtained from the

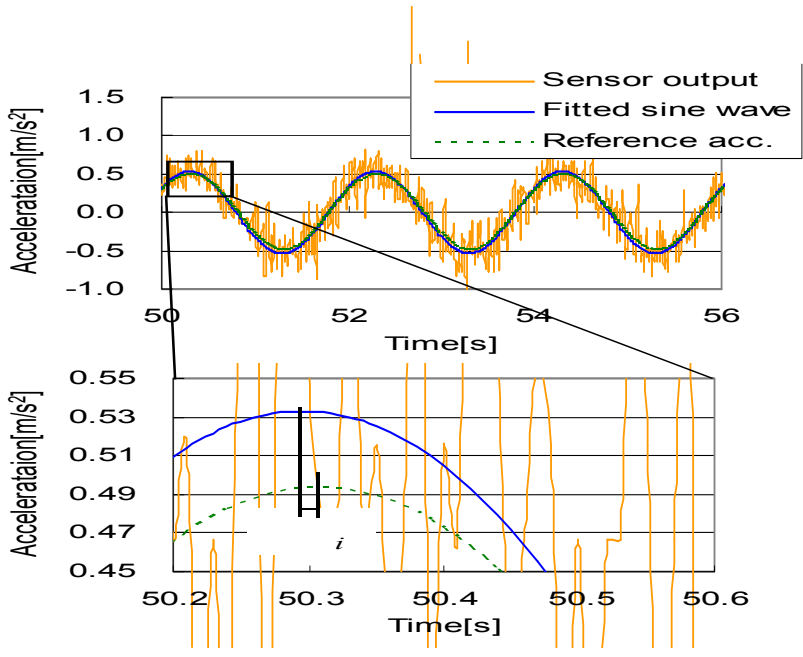

Figure 6: Sensor including planes and actual sensor direction.

corrected accelerometer can be used as velocity and displacement with dead reckoning. Especially, displacement data of large building are quite important information for the safety inspection.

\section{A New Calibration Method}

Figure 2 illustrates our new calibration method of an accelerometer. This method uses an exciter mechanism of parallel linkage with balanced structure. The exciter device generates accurate and stable uniform circular motion. The input acceleration is reliable sine wave for each sensor.

Almost all conventional calibration methods adopt a linear motion, however it costs to keep the accuracy. Moreover, it cannot distinguish the sensitivity and the inclination of sensor direction. In contrast, the proposed method can measure both sensor direction and sensitivity as denoted in figure 3 . Figure 4 shows the exciter device with parallel linkage.

This device provides three sets of sine wave output voltages from 3 -axis accelerometer. The output provides three phase angles at the maximum voltages. These data mean three planes which include actual sensor directions (Figure 5). Two times measurement in different sensor postures gives six planes. The actual sensor direction is obtained from the line of intersection of the corresponding two planes. From the information of sensor direction, the sensitivity is calculated from the amplitude of the sine wave output. Figure 6 shows an example of measurement data with a commercially available accelerometer. Because it is generic product, the output signal includes large noise, however the proposed method can detect actual sensor directions and sensitivities. This means that the method can deal with various accelerometers, not only high accuracy type but also bad $\mathrm{S} / \mathrm{N}$ ratio type.

\section{References}

1. ISO 16063-1 (1998) Methods for the calibration of vibration and shock transducers Part 1: Basic concepts.

2. ISO 5347-13 (1993) Methods for the calibration of vibration and shock pick-ups Part 13: Testing of base strain sensitivity.

3. A Umeda (2007) Calibration of Three-axis Accelerometers as a ThreeDimensional Accelerometer Using a Three-Dimensional Vibration Generato and Laser Interferometers : Proposal of the New Technique from the Viewpoint that the Acceleration is a Vector Quantity", Transactions of the JSME 70: 38-45. 